\title{
, \\ BHMPS Inhibits Breast Cancer Migration and Invasion by Disrupting Rab27a-Mediated EGFR and Fibronectin Secretion
}

\author{
Jeong-In Park ${ }^{1,2}$, Kyung-Hee Song ${ }^{1}\left(\right.$, , Seong-Mook Kang ${ }^{1}$, Jeeyong Lee ${ }^{1}$, Seong-Jun Cho ${ }^{3}$, Hyun Kyung Choi ${ }^{4}$, \\ Jiyeon Ahn ${ }^{1}{ }^{\circledR}$, Jong-Kuk Park ${ }^{1}$, Jaesung Kim ${ }^{1} \oplus$, Sang-Gu Hwang ${ }^{1}$, Dae-Seog Lim ${ }^{5}$, Joon Kim ${ }^{2}$, \\ Seung-Youn Jung $1, *(\mathbb{D}$ and Jie-Young Song $1, *(\mathbb{D})$
}

Citation: Park, J.-I.; Song, K.-H.; Kang, S.-M.; Lee, J.; Cho, S.-J.; Choi, H.K.; Ahn, J.; Park, J.-K.; Kim, J.; Hwang, S.-G.; et al. BHMPS Inhibits Breast Cancer Migration and Invasion by Disrupting Rab27a-Mediated EGFR and Fibronectin Secretion. Cancers 2022 14, 373. https://doi.org/10.3390/ cancers14020373

Academic Editors: Suresh K Alahari and Fabio Carraro

Received: 8 November 2021

Accepted: 10 January 2022

Published: 12 January 2022

Publisher's Note: MDPI stays neutral with regard to jurisdictional claims in published maps and institutional affiliations.

Copyright: (C) 2022 by the authors. Licensee MDPI, Basel, Switzerland. This article is an open access article distributed under the terms and conditions of the Creative Commons Attribution (CC BY) license (https:// creativecommons.org/licenses/by/ $4.0 /)$.
1 Division of Radiation Biomedical Research, Korea Institute of Radiological \& Medical Sciences, Seoul 01812, Korea; jipark@kirams.re.kr (J.-I.P.); songkh@kirams.re.kr (K.-H.S.); tjdanranr@naver.com (S.-M.K.); jeeyongl@kirams.re.kr (J.L.); ahnjy@kirams.re.kr (J.A.); jkpark@kirams.re.kr (J.-K.P.); jaesung@kirams.re.kr (J.K.); sgh63@kirams.re.kr (S.-G.H.)

2 Laboratory of Biochemistry, Division of Life Sciences, Korea University, Seoul 02841, Korea; joonkim@korea.ac.kr

3 Low-dose Radiation Research Team, Radiation Health Institute, Korea Hydro \& Nuclear Power Co., Ltd., Seoul 01450, Korea; seongjcho@khnp.co.kr

4 Department of Chemistry, Sogang University, Seoul 04107, Korea; hkchoi45@sogang.ac.kr

5 Department of Biotechnology, CHA University, Seongnam 13488, Gyeonggi-do, Korea; dslim@cha.ac.kr

* Correspondence: seungyoun@kirams.re.kr (S.-Y.J.); immu@kirams.re.kr (J.-Y.S.)

Simple Summary: Numerous studies targeting Rab GTPases and its multiple effectors have been attempted since exocytosis has been shown to alter tumor malignancy by modulating cancer cell behavior and tumor microenvironment. Here, we demonstrated that BHMPS inhibits migration and invasion of breast cancer cells by blocking the interaction between Rab27a and Slp4. BHMPS interfered with vesicle trafficking and secretion by decreasing FAK and JNK activation. In addition, BHMPS suppressed tumor growth in Rab27a-overexpressing MDA-MB-231 xenograft mice. This study highlighted the importance of understanding the mechanisms of Rab27a-mediated metastasis in improving the therapeutic options for metastatic cancers.

\begin{abstract}
Our previous work demonstrated that (E)-N-benzyl-6-(2-(3, 4-dihydroxybenzylidene) hydrazinyl)- $N$-methylpyridine-3-sulfonamide (BHMPS), a novel synthetic inhibitor of Rab27aSlp(s) interaction, suppresses tumor cell invasion and metastasis. Here, we aimed to further investigate the mechanisms of action and biological significance of BHMPS. BHMPS decreased the expression of epithelial-mesenchymal transition transcription factors through inhibition of focal adhesion kinase and c-Jun $\mathrm{N}$-terminal kinase activation, thereby reducing the migration and invasion of breast cancer. Additionally, knockdown of Rab27a inhibited tumor migration, with changes in related signaling molecules, whereas overexpression of Rab27a reversed this phenomenon. BHMPS effectively prevented the interaction of Rab27a and its effector Slp4, which was verified by co-localization, immunoprecipitation, and in situ proximity ligation assays. BHMPS decreased the secretion of epidermal growth factor receptor and fibronectin by interfering with vesicle trafficking, as indicated by increased perinuclear accumulation of CD63-positive vesicles. Moreover, administration of BHMPS suppressed tumor growth in Rab27a-overexpressing MDA-MB-231 xenograft mice. These findings suggest that BHMPS may be a promising candidate for attenuating tumor migration and invasion by blocking Rab27a-mediated exocytosis.
\end{abstract}

Keywords: Rab GTPase; invasion; migration; vesicle trafficking; breast cancer

\section{Introduction}

Metastasis is a crucial process in cancer development and is considered a leading cause of cancer therapy failure; however, its control remains a challenge. Primary cancer 
develops into secondary cancer through metastasis, which includes five key steps: invasion and migration, intravasation, circulation, extravasation, and colonization [1]. Invasion and migration, the first step of metastasis, are commonly referred to as epithelial-mesenchymal transition (EMT) and are considered a major target for cancer therapy. EMT is a biological process by which epithelial cells are transformed into a mesenchymal state and is strongly associated with the development toward an advanced cancer phenotype. During this process, cancer cells release several factors, including growth factors, chemokines, cytokines, and exosomes, to create a tumor microenvironment (TME) favorable for their survival [1,2].

Rab GTPases constitute a large family of small GTPases that regulate membrane identity and vesicle budding, uncoating, motility, and fusion, through complexing with effector proteins such as sorting adaptors, tethering factors, kinases, and phosphatases. These Rab proteins primarily mediate the trafficking of proteins and organelles involved in cell growth, survival, and motility [3,4]. Rab27a is expressed in a subset of specialized secretory cell types and modulates melanosome trafficking in melanocytes by forming a complex with the Rab27a-specific effector melanophilin (Mlph) and the motor protein myosin-Va [5]. Additionally, among the effectors, synaptotagmin-like protein 4 (Slp4) promotes the docking of multivesicular bodies (MVBs) in the plasma membrane by interacting with Rab27a [6]. Furthermore, Rab27a regulates exosome secretion to create a metastatic niche in melanoma and breast cancers $[7,8]$. Exosomes are spherical, bilayered proteolipids that enclose various molecules, such as proteins, lipids, metabolites, and nucleic acids, which are specifically organized according to the cells and effectively transmit information to the extracellular matrix (ECM) and surrounding cells $[9,10]$. Particularly, tumor-derived exosomes have been associated with the development of cancer malignancy by promoting cancer cell proliferation, establishing a premetastatic niche, causing ECM degradation, modulating immune cells and stromal cells, and regulating drug resistance [1,9,11]. Altered expression of Rab27 is observed in various human cancers, but the function or mechanism of Rab27 in cancer is not fully understood. Therefore, many researchers have focused on targeting vesicle trafficking and exocytosis pathways because blocking exocytosis may provide novel therapeutic interventions, as it inhibits metastatic cancer and improves cancer therapeutic efficacy [12].

Initially, BMD-11, the BHMPS parent compound, was isolated as a potent proteinprotein inhibitor at the binding interface between Rab27a and melanophilin through virtual screening to discover novel melanosome transport inhibitors for functional cosmetics [13]. Since then, we have synthesized 15 BMD-11 derivatives and selected (E)-N-benzyl-6(2-(3,4-dihydroxy benzylidene)hydrazinyl)-N-methylpyridine-3-sulfonamide (BHMPS), a new Rab27a-targeting synthetic compound that inhibits migration and invasion by downregulating ECM marker levels in A375 melanoma and MDA-MB-231 breast cancer cells [14]. However, the underlying mechanisms remain largely unknown. Therefore, this study further investigated the antimetastatic potential of BHMPS by focusing on Rab27a-mediated signaling pathways.

\section{Results}

\subsection{BHMPS Suppresses Migration and Invasion of Breast Cancer Cells}

To confirm the antimetastatic potential of BHMPS, a new synthetic compound that can inhibit Rab27a expression [14], we first verified Rab27a expression in breast cancer cell lines with different subtypes, including MDA-MB-231 and BT549 for TNBC, BT474 for luminal $\mathrm{B}$, and MCF7 for luminal A molecular subtypes. Intrinsic expression of Rab27a protein was higher in MDA-MB-231 and BT549 cells than in BT474 and MCF7 cells (Figure 1A). Moreover, serum stimulation further increased Rab27a expression in the TNBC cells. Next, the effect of BHMPS on cell viability was evaluated using the MTT assay (Figure 1B). MCF7 cells showed the lowest $50 \%$ growth inhibition $\left(\mathrm{GI}_{50}\right)$ value of $18.62 \mu \mathrm{M}$, whereas BHMPS below $10 \mu \mathrm{M}$ did not inhibit the growth of other breast cancer cells. Treatment of BHMPS at a non-cytotoxic dose of $10 \mu \mathrm{M}$ significantly inhibited the migratory and invasive ability of MDA-MB-231 and BT549 cells with high expression of Rab27a (Figure 1C,D). 
A
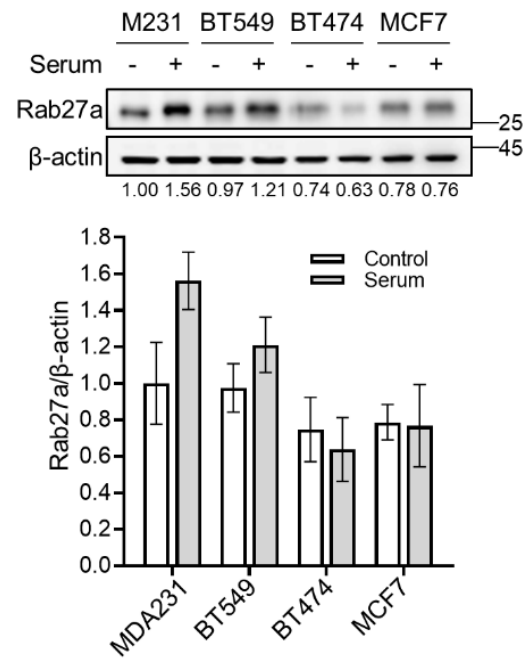

C
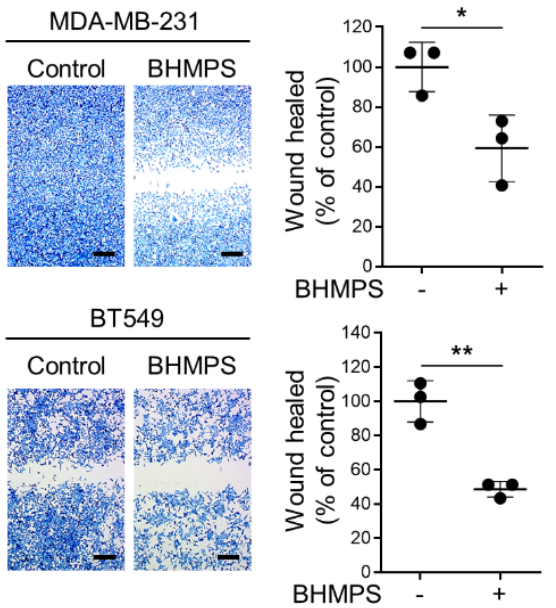
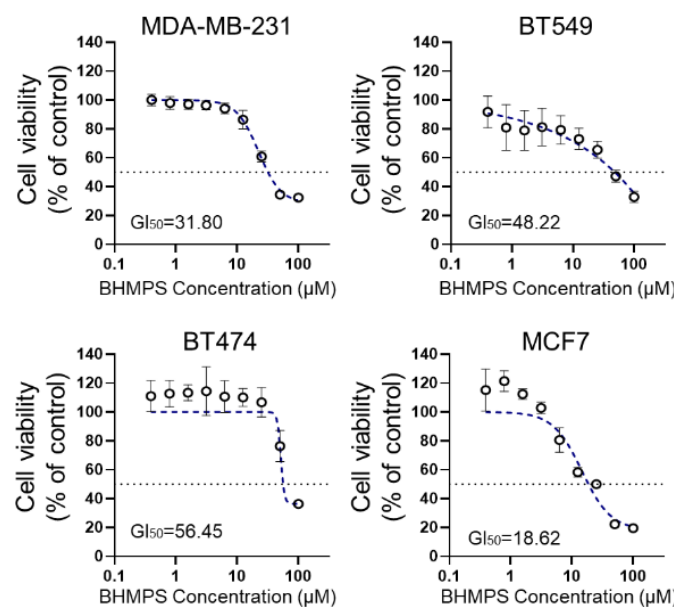

D
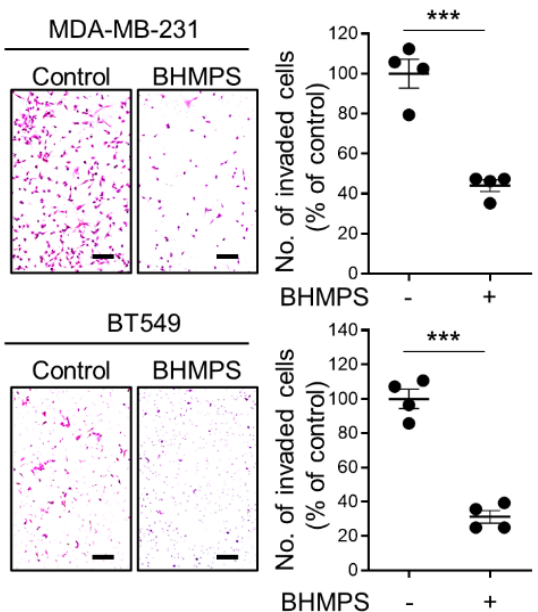

Figure 1. BHMPS inhibits migration and invasion in breast cancer cells. (A) Basal levels of Rab27a in several breast cancer cell lines were identified using western blotting. The uncropped images are shown in Supplementary Materials. Relative Rab27a levels were quantified using densitometry with ImageJ software, normalized to $\beta$-actin level, and expressed as fold-change compared to that of the MDA-MB-231 control. Data represent the average of three independent experiments. (B) MTT assays were performed to determine cell viability. Cells were treated with the indicated concentrations of BHMPS $(0-100 \mu \mathrm{M})$ for $24 \mathrm{~h}$. Data shown are the means \pm SD of three independent experiments. (C) MDA-MB-231 and BT549 cells were seeded into 6-well plates and scratched using a $200 \mu \mathrm{L}$ pipette tip. The cells were treated with $10 \mu \mathrm{M}$ of BHMPS for $24 \mathrm{~h}$, after which the cells were stained and photographed using a microscope at $40 \times$ magnification (Scale bars $250 \mu \mathrm{m}$ ). Percentage wound healing was quantified by measuring the distance of the wound. Data shown are means \pm SD of three independent experiments; ${ }^{*} p<0.05$ and ${ }^{* *} p<0.01$ compared with the control. (D) MDA-MB-231 and BT549 cells were seeded in a Matrigel-coated upper chamber. The lower chamber was treated with $10 \mu \mathrm{M}$ of BHMPS, and the cells were allowed to invade through a Transwell membrane for $24 \mathrm{~h}$. The cells were stained and photographed using a microscope at a magnification of $40 \times$ (Scale bars $250 \mu \mathrm{m})$. The invaded cells were counted in five randomly selected regions in each experiment. Data shown are means $\pm \mathrm{SD}$ of four independent experiments; ${ }^{* * *} p<0.001$ compared with the control.

\subsection{BHMPS or Rab27a Silencing Decreases Activation of FAK and JNK}

To investigate the underlying mechanism of the antimetastatic potential of BHMPS, several signaling molecules involved in invasion and metastasis were examined. BHMPS inhibited the expression of target molecule Rab27a in MDA-MB-231 and BT549 cells (Figure 2A). 
Additionally, BHMPS effectively decreased the levels of serum-stimulated EMT-activating transcription factors, including Snail, Slug, Zeb1, and Twist1, which are highly related to cancer migration and invasion [15]. Consistent with previous findings that FAK and mitogen-activated protein kinase (MAPK) signaling play essential roles in cancer cell proliferation and metastasis [16,17], FAK, JNK, p38, and ERK1/2 phosphorylation was significantly increased by serum stimulation in both cells, and BHMPS treatment markedly inhibited the phosphorylation of these molecules, especially FAK and JNK (Figure 2B).

When Rab27a expression was suppressed with gene-specific siRNA, the migration of both TNBC cells was inhibited to a similar degree as that of the control without serum stimulation (Figure 2C). Rab27a knockdown also reduced FAK and JNK phosphorylation but did not alter pp38 and pERK1/2 expression in either cell line (Figure 2D).

A

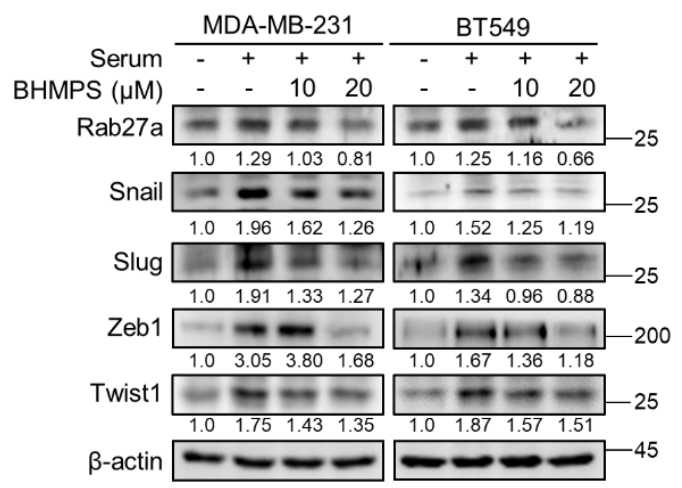

B

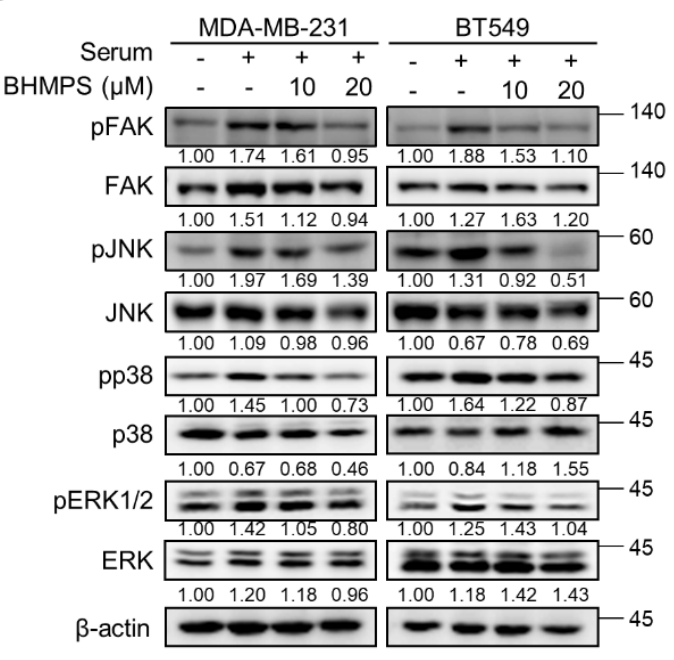

D

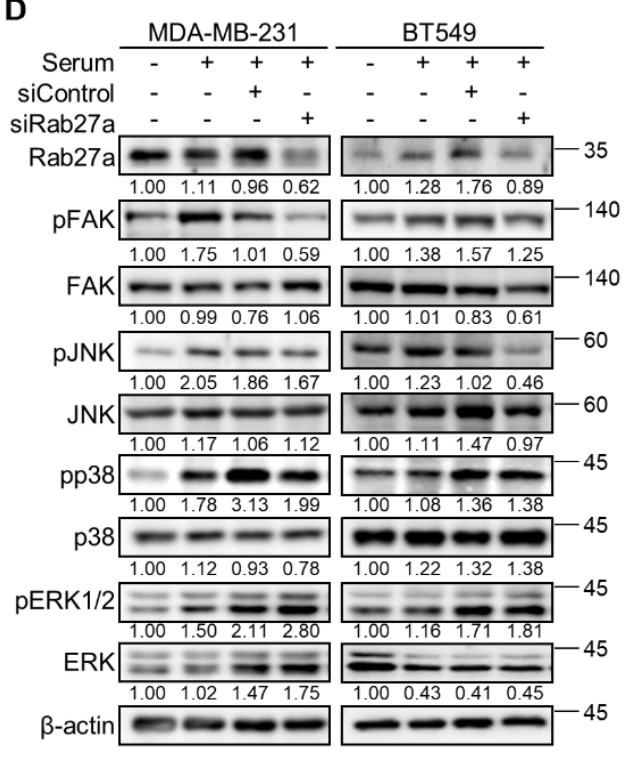

$\beta$ actin $=-$
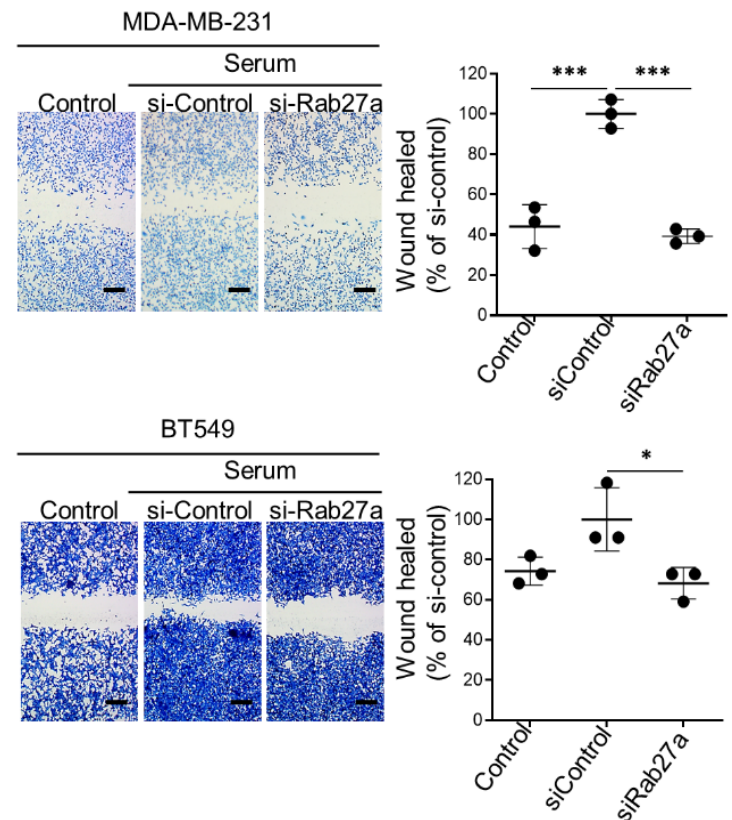
(C) MDA-MB-231 and BT549 cells were transfected with control siRNA (siControl) or siRab27a. After $24 \mathrm{~h}$, the cells were scratched, and the percentage of migrated cells was determined at $24 \mathrm{~h}$ post-scratch. Statistically significant differences are shown in comparison with siControl-transfected cells. Representative images $(40 \times$ magnification, Scale bars $250 \mu \mathrm{m})$ and means $\pm \mathrm{SD}$ are from three independent experiments. ${ }^{*} p<0.05$ and ${ }^{* * *} p<0.001$ compared with siControl-transfected cells. (D) Overnight serum-starved cells were stimulated with serum and the indicated target of siRNA for $24 \mathrm{~h}$. FAK and MAPK phosphorylation was determined by western blotting $24 \mathrm{~h}$ after transfection. The uncropped images are shown in Supplementary Materials.

To further confirm the role of Rab27a and BHMPS in cell migration, Rab27a overexpression was induced in breast cancer cells with low Rab27a expression levels. Rab27a overexpression significantly enhanced cell migration and increased expression of EMTactivating transcription factors in BT474 and MCF7 cells compared with the controls; however, BHMPS treatment effectively suppressed these effects (Figure 3A,B). Additionally, treatment with BHMPS reduced FAK and JNK phosphorylation increased by Rab27a overexpression in both cells (Figure 3C). When Rab27a-overexpressing cells were treated with JNK (SP600125) or FAK (PF573228) inhibitor, SP600125 suppressed FAK phosphorylation but PF573228 did not block JNK phosphorylation, indicating that JNK is an upstream signaling molecule of FAK in Rab27a-overexpressing cells (Figure 3D,E). Taken together, these results suggest that BHMPS suppressed the migration of breast cancer cells by inhibiting Rab27a expression, which transmitted signals to JNK and FAK activation.

A
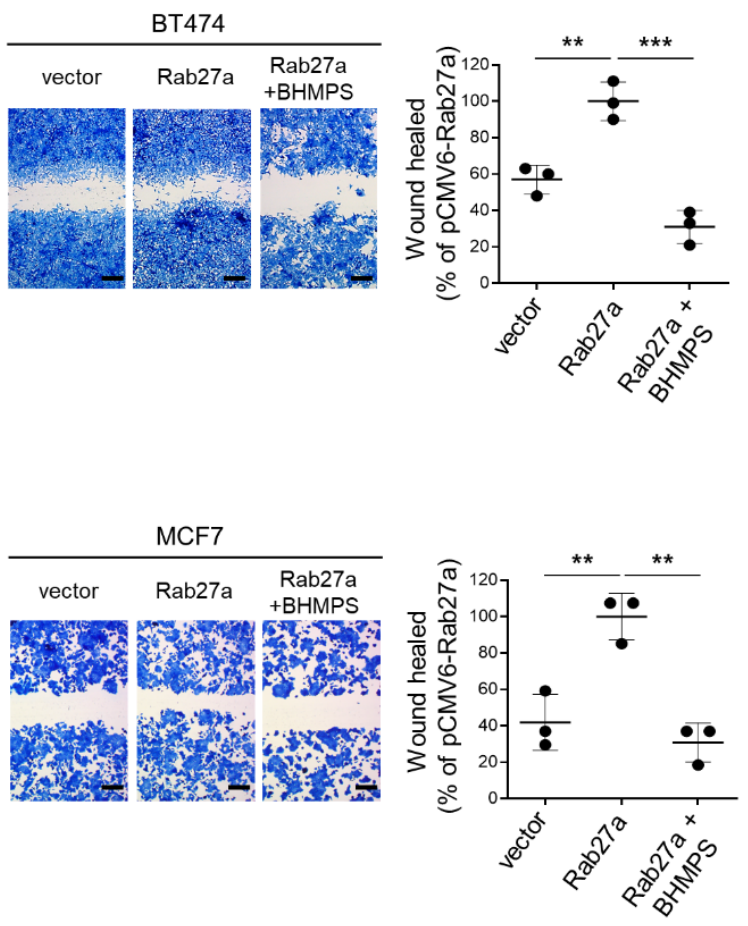

B

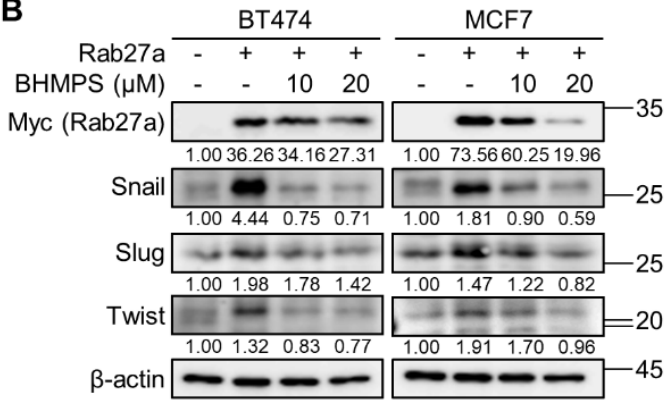

C

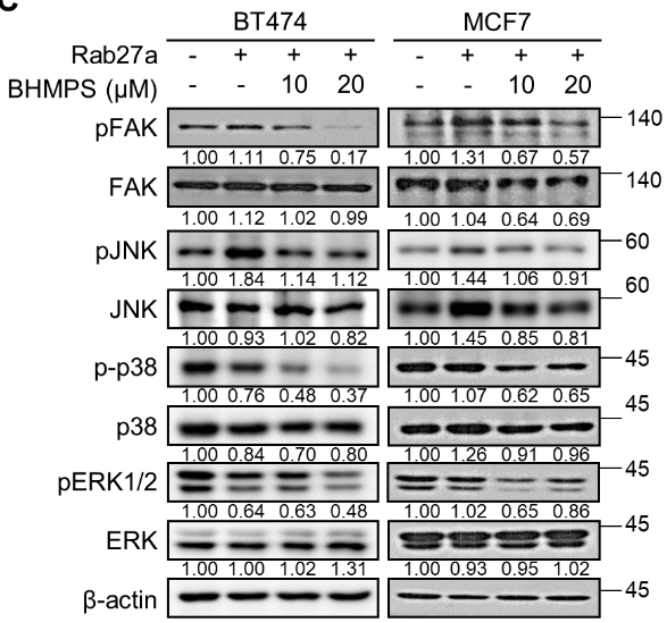

Figure 3. Cont. 
D

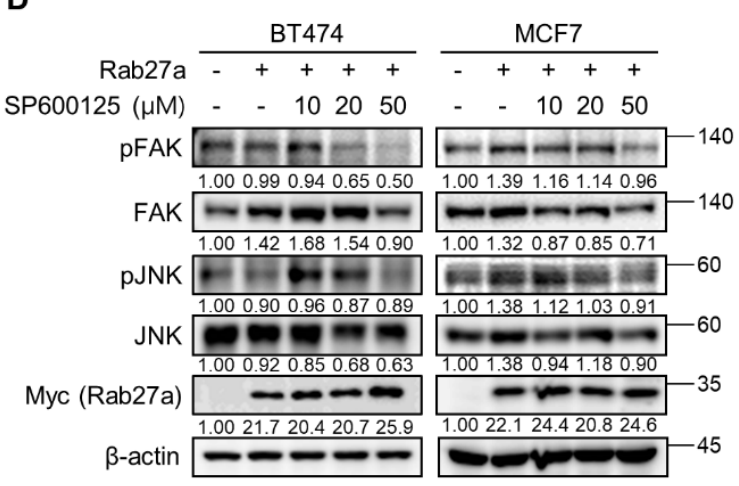

E

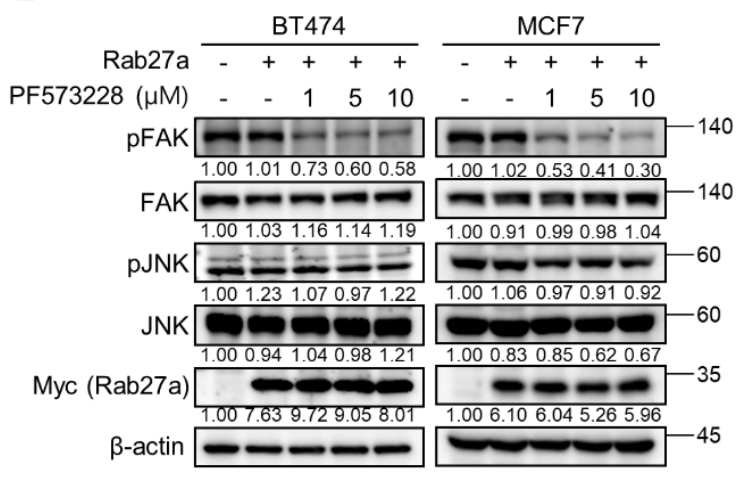

Figure 3. Rab27a overexpression promotes breast cancer cell migration. (A) BT474 and MCF7 cells were transfected with control or Rab27a expression vector. After $24 \mathrm{~h}$, the cells were treated with $10 \mu \mathrm{M}$ of BHMPS, and the percentage of migrated cells was determined. Statistically significant differences are shown in comparison with the Rab27a overexpression group. Representative images $(40 \times$ magnification, Scale bars $250 \mu \mathrm{m})$ and means \pm SD are from three independent experiments. ** $p<0.01$ and ${ }^{* * *} p<0.001$ compared with Rab27a overexpression control. (B) BT474 and MCF7 cells were transfected with Rab27a-Myc-DDK expression vector. After $24 \mathrm{~h}$, the cells were treated with the indicated concentrations of BHMPS for $24 \mathrm{~h}$. The immunoblots show the expression of EMT-activating transcription factors. (C) Under the same experimental conditions as (B), FAK and MAPK signaling was determined by western blotting. (D,E) After $24 \mathrm{~h}$ of Rab27a overexpression, cells were treated with the indicated concentrations of JNK inhibitor (SP600125) or FAK inhibitor (PF573228) for $4 \mathrm{~h}$. The uncropped images are shown in Supplementary Materials.

\subsection{BHMPS Inhibits the Interaction of Rab27a with Slp4}

Rab27a can bind to multiple factors and interact with specific effectors tailored to a particular biological setting [18]. Given that Rab27a interacts with Slp4 and Mlph in breast cancer cells [19], the expression of the two effector molecules was examined. In MDA-MB-231 and BT549 cells, serum stimulation significantly increased the expression of Slp4 and Mlph, along with that of Rab27a. However, BHMPS significantly decreased the expression of Slp4 in a dose-dependent manner compared with that of Mlph (Figure 4A). Therefore, we further examined the interaction between Rab27a and Slp4. As shown in Figure 4B, serum stimulation increased the expression of both Rab27a and Slp4 and their co-localization. Additionally, the number of PLA foci visualized as red fluorescent dots was increased by serum stimulation, indicating that there is an interaction between Rab27a and Slp4. As expected, BHMPS treatment reduced this phenomenon (Figure 4C). To confirm the direct interaction between Rab27a and Slp4, immunoprecipitation was performed using Rab27a-overexpressing MDA-MB-231 cells because the intrinsic levels of Rab27a were insufficient to detect molecular binding. BHMPS treatment decreased the precipitation of Slp4 compared with that of Rab27a-Myc, indicating that BHMPS inhibited the binding of Rab27a and Slp4 in breast cancer cells (Figure 4D). 


\section{A}

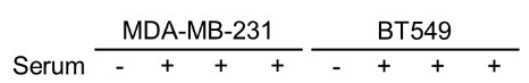
BHMPS $(\mu \mathrm{M}) \quad-\quad-\quad 10 \quad 20 \quad-\quad-10 \quad 20$

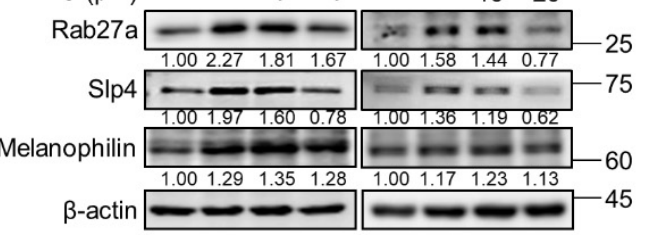

C
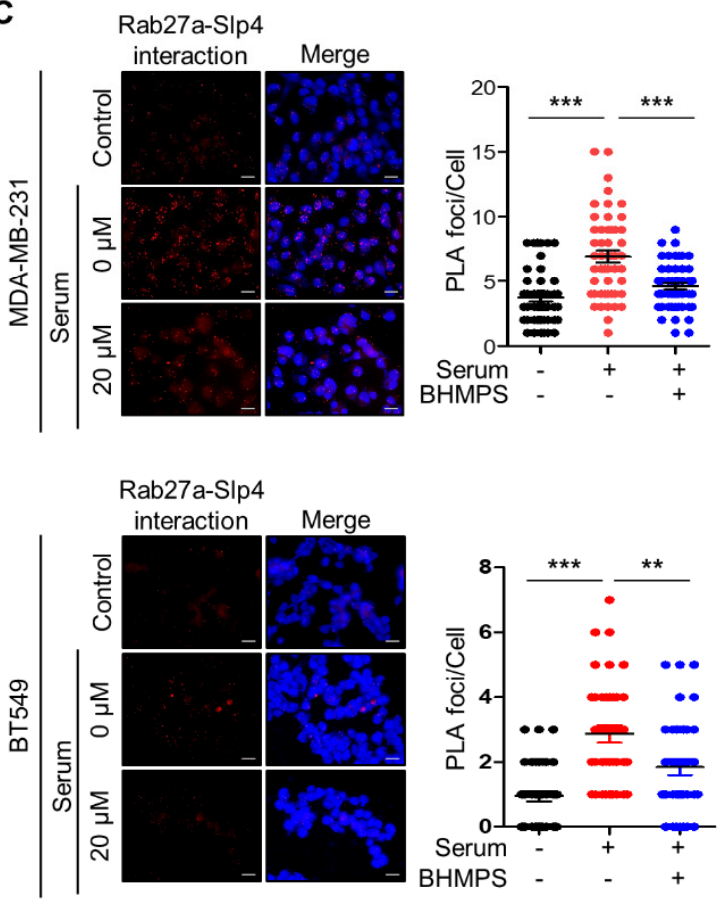

B

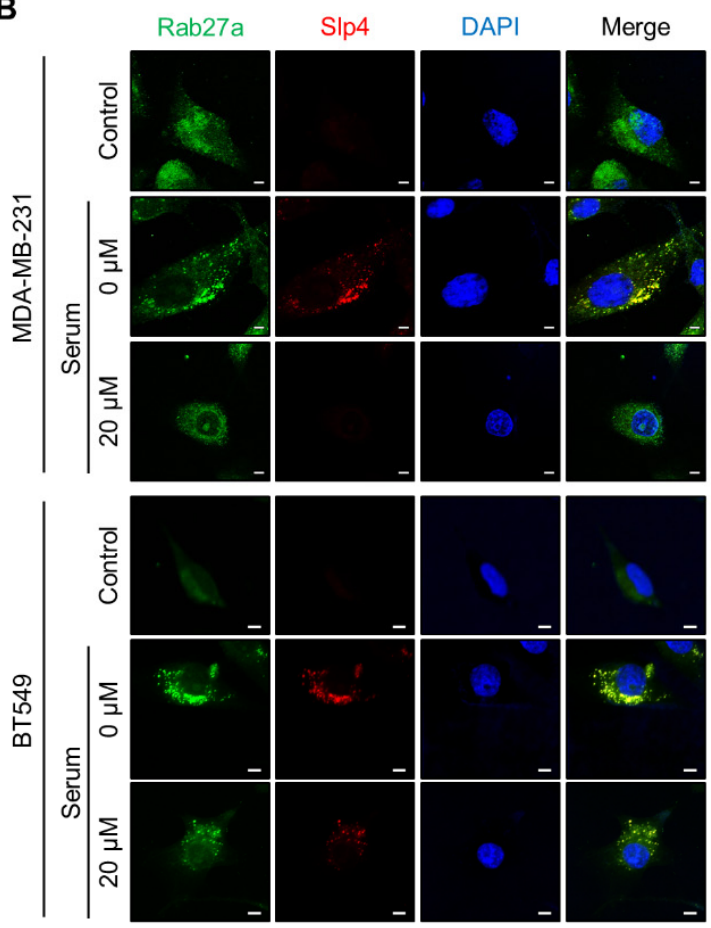

D

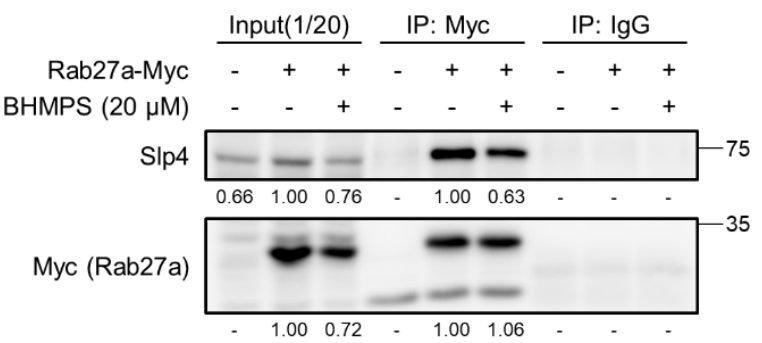

Figure 4. BHMPS disrupts Rab27a and Slp4 interaction. (A) Overnight serum-starved cells were stimulated with $10 \%$ FBS and co-treated with the indicated concentrations of BHMPS for $24 \mathrm{~h}$. The levels of indicated proteins were measured using western blotting. (B) The expression levels of Rab27a (Green) and Slp4 (Red) were detected using specific monoclonal antibodies and an Alexa 488- or 594-conjugated secondary antibody, respectively. Nuclei were stained with DAPI (Blue), and samples were analyzed using confocal microscopy. Representative images from three independent experiments are shown (Scale bars $5 \mu \mathrm{m}$ ). (C) Under the same experimental conditions as (A), Duolink $^{\mathrm{TM}}$ in situ PLA was performed using MDA-MB-231 and BT549 cells. For the assay, both rabbit anti-Rab27a and mouse anti-Slp4 antibodies were used. The interactions $(<40 \mathrm{~nm})$ of Rab27a with Slp4 are represented as red dots (Scale bars $20 \mu \mathrm{m}$ ), which were counted in randomly selected regions in each experiment. Nuclei were counterstained with DAPI (Blue). Nuclear foci were counted from at least 50 nuclei. Data are shown as the mean $\pm \mathrm{SD}$ of the red dots counted per nucleus. ${ }^{* *} p<0.01$ and ${ }^{* * *} p<0.001$ compared with serum stimulation. (D) Rab27a-Myc-DDK-stably expressing MDAMB-231 cell was produced to verify that BHMPS can inhibit the interaction between Rab27a and Slp4. Protein samples $(1000 \mu \mathrm{g})$ were immunoprecipitated with a magnetic bead-conjugated Myc-tag mouse monoclonal antibody or mouse normal IgG used as a negative control. Values are expressed as the average intensity of each protein compared to the untreated sample from three independent experiments. The uncropped images are shown in Supplementary Materials.

\subsection{BHMPS Interferes with Vesicle Trafficking and Secretion}

Considering the role of Rab27a in vesicle transport and exocytosis, the intracellular localization of Rab27a and CD63 was examined. A member of the tetraspanin superfamily, CD63, is highly enriched in late endosomal MVBs and exosomes [20]. Serum stimulation 
induced co-localization of CD63 and Rab27a near the plasma membranes of TNBC cells, while BHMPS accumulated CD63 in the perinuclear region and markedly attenuated this co-localization in the plasma membrane (Figure 5A). These results suggest that Rab27a inhibition by BHMPS could suppress the transport of vesicles to the plasma membrane and secretion. Since EGFR and FN are considered clinically relevant exosome protein markers for breast cancer [21], the expression of EGFR and FN in the conditioned medium was examined. Serum stimulation significantly increased the release of EGFR and FN, and BHMPS effectively suppressed their release (Figure 5B).

A
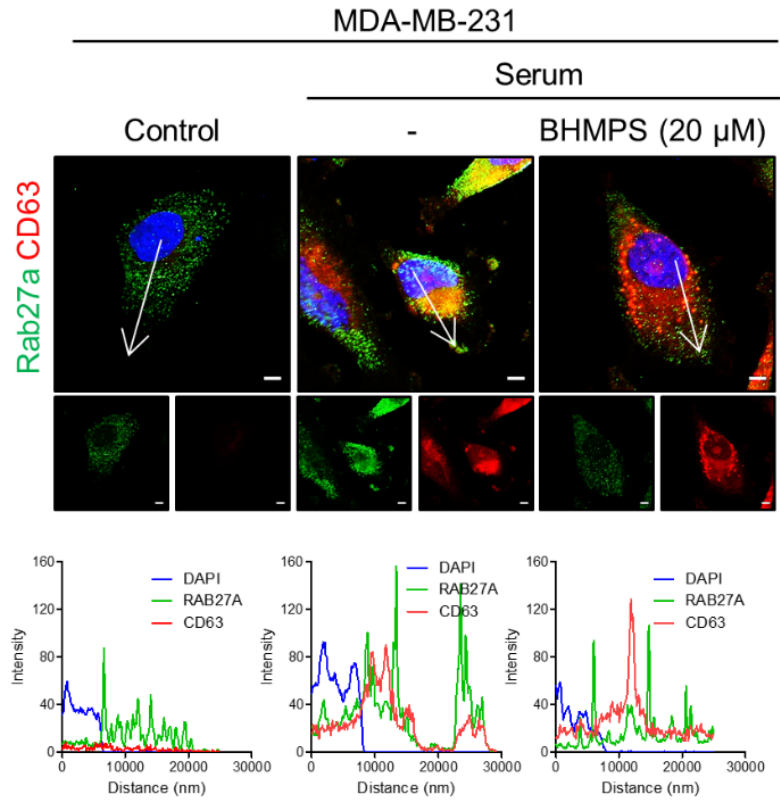

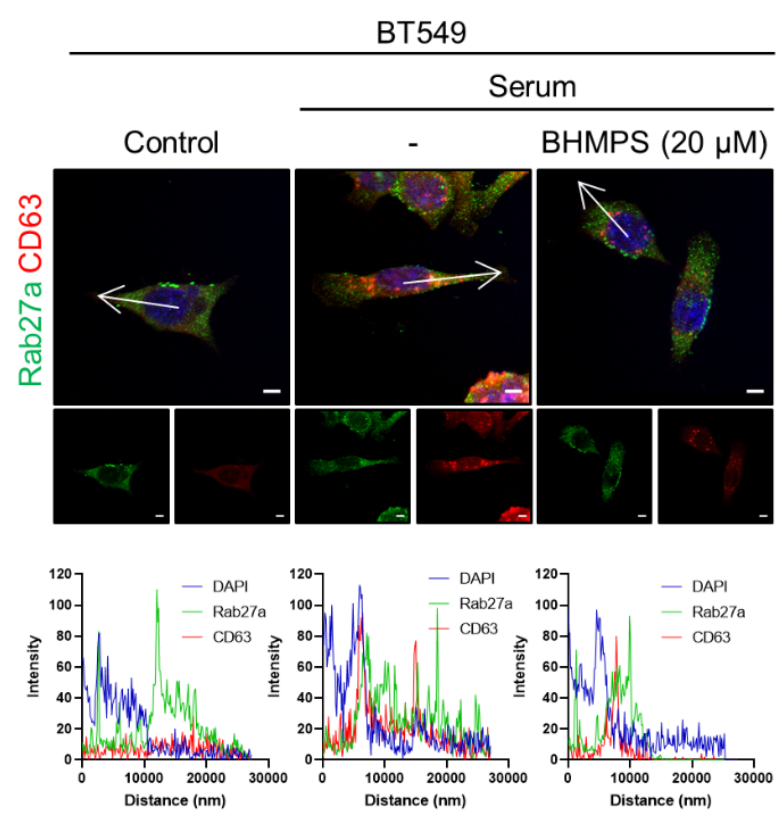

B

\begin{tabular}{|c|c|c|c|c|c|c|c|c|}
\hline & MDA- & MB-2 & & & BT5 & 549 & & \\
\hline Serum & + & + & + & - & + & + & + & \\
\hline BHMPS $(\mu \mathrm{M})$ & - & 10 & 20 & - & - & 10 & 20 & \\
\hline EGFR & 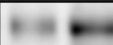 & 28 & $=0$ & & F. & 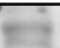 & +4 & 30 \\
\hline & $\begin{array}{lll}1.00 & 1.84\end{array}$ & 1.65 & 1.64 & 1.00 & 4.78 & 2.54 & 1.77 & \\
\hline Fibronectin & 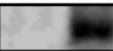 & 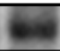 & $\sqrt{4}$ & 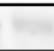 & 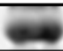 & $E$ & Wat & -245 \\
\hline & 1.004 .04 & 3.78 & 2.10 & 1.00 & 6.81 & 3.09 & 59 & \\
\hline
\end{tabular}

Figure 5. BHMPS interferes with the vesicle secretion process. (A) Confocal microscopic analysis was performed to examine the localization of exosome and Rab27a altered by BHMPS in MDA-MB-231 and BT549 cells. Exosome (Red) was labeled with the CD63 antibody and an Alexa 594-conjugated secondary antibody. Rab27a (Green) was labeled with the Rab27a antibody and an Alexa 488conjugated secondary antibody. DAPI $(300 \mathrm{ng} / \mathrm{mL})$ was used to mark the nucleus (Blue). The scale bar represents $5 \mu \mathrm{m}$. The single channel images of Rab27a and CD63 are displayed below the left and right of the merged image, respectively. The fluorescence intensities at the marked white arrow were analyzed using Carl Zeiss ZEN lite software and represented in the bottom panel. (B) Overnight serum-starved cells were stimulated with 10\% FBS and co-treated with the indicated concentrations of BHMPS for $24 \mathrm{~h}$, and then conditioned media were collected. After quantification, levels of the specified proteins were analyzed using western blotting with the same concentration of proteins. The uncropped blots and the images of Ponceau S-stained membrane are shown in Supplementary Materials. 


\subsection{BHMPS Inhibits Tumor Growth in Rab27a-Overexpressing Tumor Xenograft Mice}

To investigate whether the above results could be replicated in vivo, the antitumor effect of BHMPS was evaluated in an experimental lung metastasis model of B16F10 melanoma. BHMPS administration did not show any antimetastatic effects (Figure 6A-C). Similarly, tumor growth or metastasis was not affected by BHMPS in the MDA-MD-231 cell xenograft mouse model and the spontaneous metastasis model of 4T1/luc cells (data not shown). Therefore, we further examined tumor growth in Rab27a-overexpressing MDA-MB-231 cell xenograft mice with elevated levels of Rab27a. The viability of Rab27aoverexpressed cells was similar to that of the control parental cells, and the $\mathrm{GI}_{50}$ value of BHMPS did not differ between the two cells (Figure 6D,E). Interestingly, tumor growth in mice transplanted with Rab27a-overexpressing cells was significantly delayed compared to that of the parental control cells, and BHMPS was shown to inhibit tumor growth only in Rab27a-overexpressing tumors (Figure 6F,G). To determine whether this phenomenon is related to tumor immunity, a complete blood count (CBC) analysis was performed. The number of monocytes and neutrophils were significantly decreased in Rab27a-overexpressing tumor xenograft mice compared to the control group (Figure $6 \mathrm{H}$ ). The number of lymphocytes, red blood cells and platelets was not different between the two groups.

A

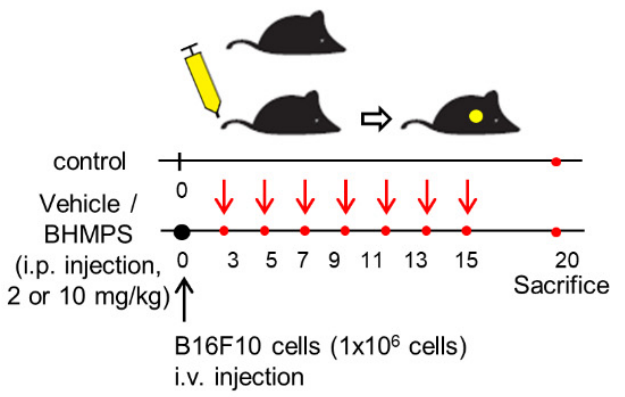

B

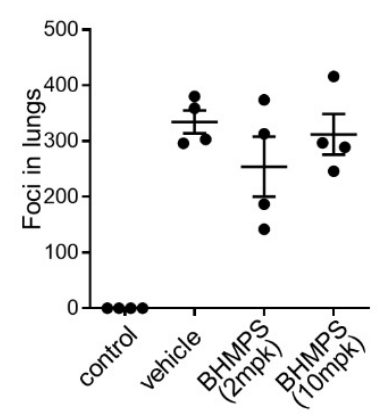

C

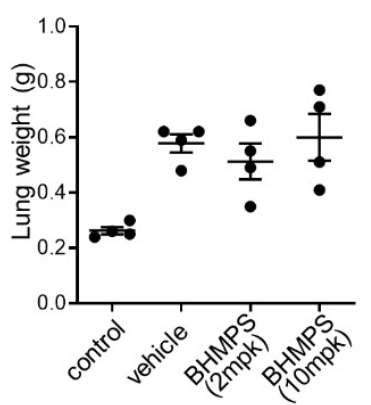

D

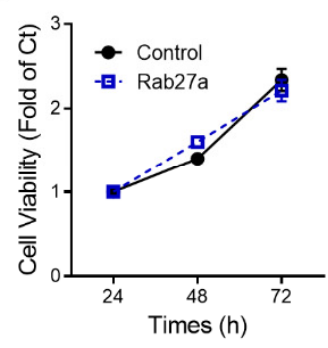

E

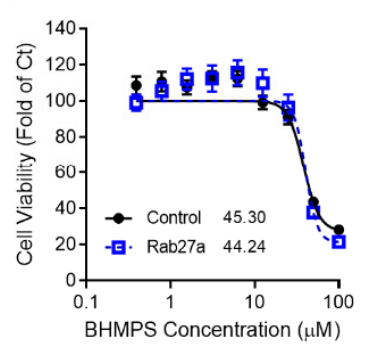

F

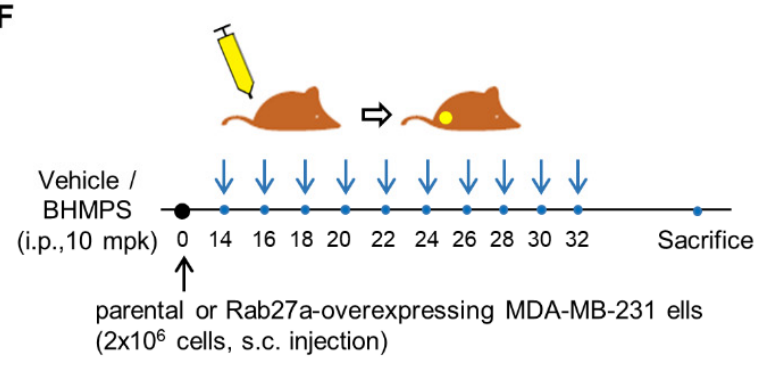

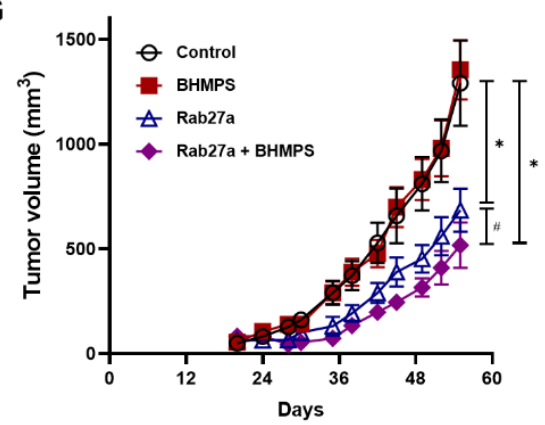

H

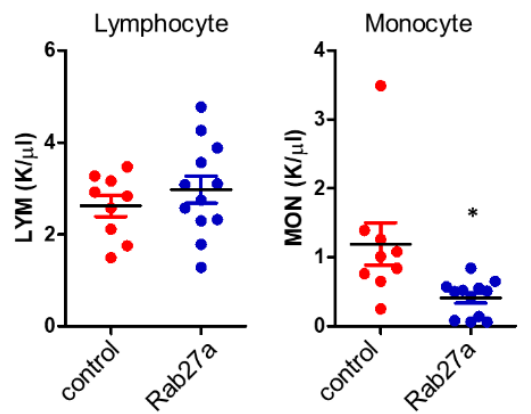

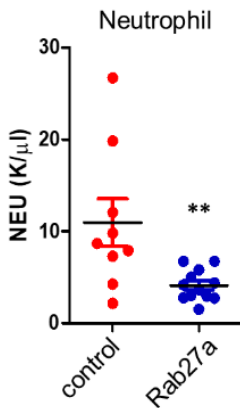

Figure 6. BHMPS suppresses the growth of Rab27a-overexpressed tumor. (A) Schematic diagram of in vivo BHMPS treatment in the experimental lung metastasis model. (B) B16F10 cells $\left(1 \times 10^{6}\right.$ cells $/$ mouse) were injected intravenously into the tail vein of C57BL/ 6 mice ( $n=4$ mice/group). 
Three days after tumor cell injection, BHMPS (2 or $10 \mathrm{mg} / \mathrm{kg}$ ) or vehicle was administrated intraperitoneally every $2 \mathrm{~d}$ for a total of six times. After mouse sacrifice, numbers of cell colonies metastasized to the lungs were counted and the mean \pm SE are shown. (C) Data shown are the means \pm SE of weight of the lungs. (D) MTT assay was performed to determine the viability of Rab27a-overexpressing cells. The viability from $24 \mathrm{~h}$ to $72 \mathrm{~h}$ after seeding with the same number of cells was analyzed. Data shown are the means \pm SD of three independent experiments. (E) MTT assay was performed to determine the effect of BHMPS on the viability of Rab27a-overexpressing cells. Cells were treated with the indicated concentrations of BHMPS $(0-100 \mu \mathrm{M})$ for $24 \mathrm{~h}$. Data shown are the means \pm SD of three independent experiments. (F) Schematic of in vivo BHMPS administration in the tumor xenograft model. (G) Control (parental MDA-MB-231 cells) or Rab27a-overexpressed MDA-MB-231 cell $\left(2 \times 10^{6}\right.$ cells/mouse $)$ were injected subcutaneously into the hind thigh of BALB/c nude mice ( $n=6-8$ mice/group). Fourteen days after tumor cell injection, BHMPS $(10 \mathrm{mg} / \mathrm{kg})$ or vehicle was administrated intraperitoneally every $2 \mathrm{~d}$ for a total of 10 times. The means $\pm \mathrm{SE}$ of tumor size change over time were shown, and significance was analyzed with repeated measures one-way ANOVA and Tukey's post hoc comparisons. (H) MDA-MB-231 $(n=9)$ or Rab27a-overexpressed MDA-MB-231 cells $(n=12)$ were injected subcutaneously into the hind thigh of BALB/c nude mice. Fifty-five days after tumor cell injection, peripheral blood was collected, and CBC were analyzed. The relative levels of the indicated CBC parameters are shown. Data are shown as the mean $\pm \mathrm{SE}$. ${ }^{*} p<0.05$ and ${ }^{* *} p<0.01$ compared with control, $\# p<0.05$ compared with Rab27a-overexpressing group.

\subsection{Association between Rab27a Expression and OS of Breast Cancer Patients}

Given the conflicting results of Rab27a overexpression in in vitro and in vivo studies, we analyzed the clinical significance of Rab27a expression levels using public expression profiles. Rab27a expression was significantly higher in glioblastoma and thyroid carcinoma than in adjacent normal tissues but lower in breast cancer (Supplementary Figure S1). Investigating the association between Rab27a expression and patient survival showed that OS in breast cancer patients was longer in the high Rab27a expression group than that in the low Rab27a expression group (Figure 7A). Additionally, Rab27a expression was significantly lower in the luminal or Her-2 positive subclasses as well as in stage 2 to 4 invasive breast carcinoma than in the normal group (Figure $7 \mathrm{~B}, \mathrm{C}$ ). In contrast to the above observations, patients with high levels of Rab27a protein showed poor survival, and there was no difference in the expression of Rab27a protein between tumor stages. The expression of Rab27a was significantly higher only in the Her-2 positive subgroup than in the normal group (Figure 7D-F). The value of Rab27a as a prognostic marker in cancer patients was assessed differentially by dataset (sample size), cancer type, and analysis program. According to the Human Protein Atlas, high expression of Rab27a decreased the survival rate of renal cancer patients but increased that of breast cancer patients. Therefore, it remains controversial whether high Rab27a expression is suitable for predicting cancer prognosis. 


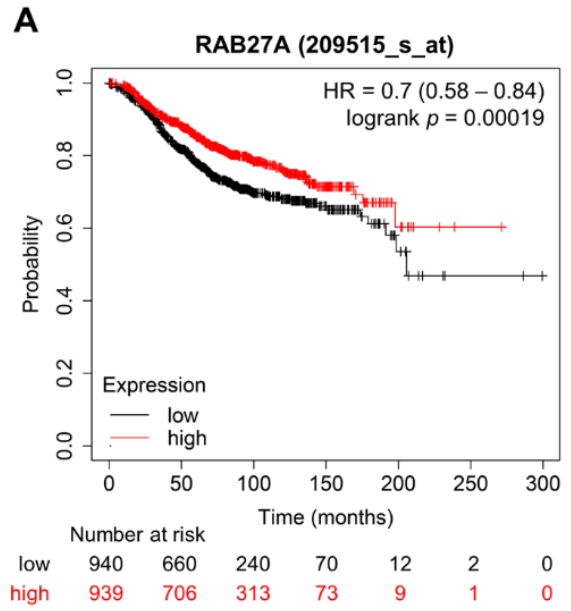

B

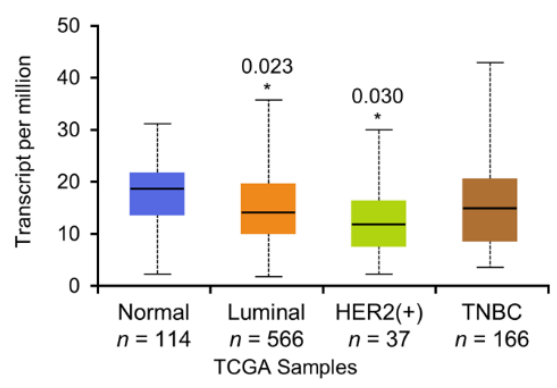

E

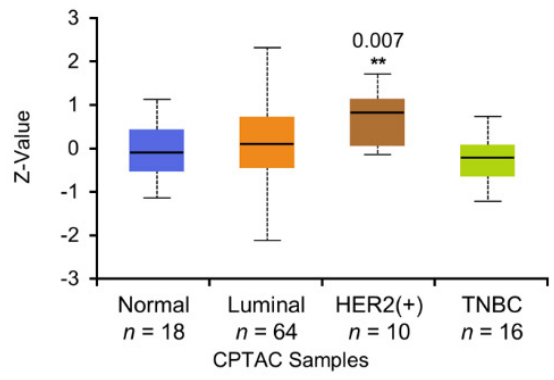

C

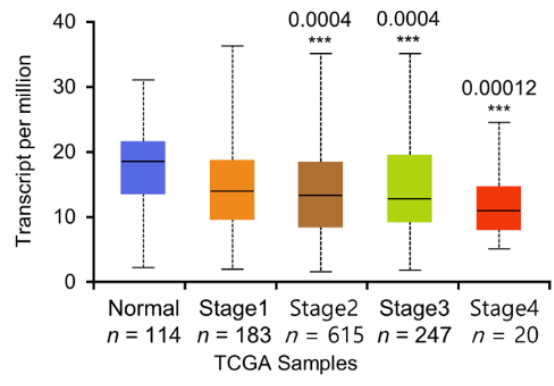

D

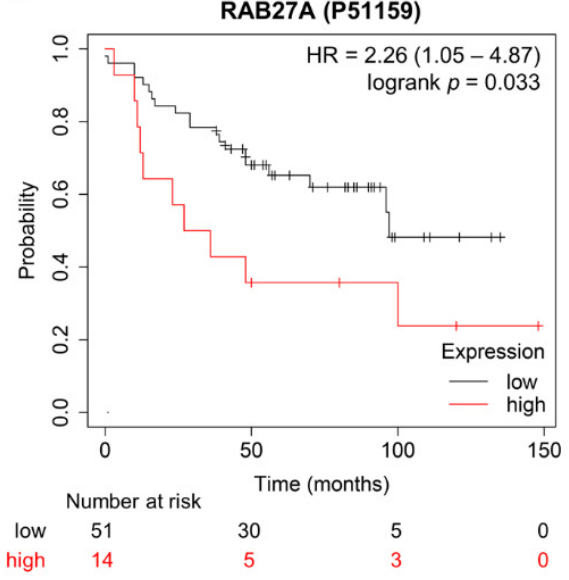

$\mathbf{F}$

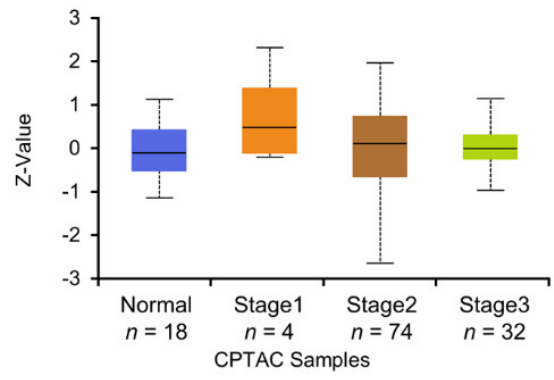

Figure 7. Clinical significance of Rab27a mRNA and protein expression levels. (A) Effect of Rab27a mRNA on OS of breast cancer patients, using Kaplan-Meier Plotter analysis. The image with the lowest logrank $p$ value is represented. The red and black lines indicate high and low Rab27a expression, respectively. (B,C) Rab27a mRNA expression levels in (B) major subclasses of breast cancer or (C) different breast cancer stages were analyzed with the UALCAN tool using TCGA BRCA dataset. (D) the overall survival rate of breast cancer patients based on Rab27a expression at the protein level was analyzed using the Kaplan-Meier Plotter. (E,F) The expression level of Rab27a protein according to (E) major subclasses or $(\mathbf{F})$ stages of breast cancer was analyzed with UALCAN tool using the CPTAC breast cancer dataset. Boxplot represents median with upper and lower quartiles, and the bars represent the maximum and minimum values. Compared with the normal tissue control group, statistically significant differences were indicated as ${ }^{*} p<0.05,{ }^{* *} p<0.01$, and *** $p<0.001$.

\section{Discussion}

We demonstrated that BHMPS could effectively inhibit the migration potential of breast cancer cells with high expression of Rab27a through down-regulation of FAK and JNK signaling pathways. FAK is a non-receptor tyrosine kinase that mediates signal transduction by integrins and other cell surface receptors to regulate cell adhesion, migration, survival, differentiation, and metastasis in a variety of cells [22,23]. FAK is known as a crucial downstream signaling molecule in the trafficking of integrin that continuously undergoes endo/exocytic trafficking to facilitate focal adhesion turnover, cell migration, invasion, and cytokinesis, resulting in altered polymerization or stabilization of actin and microtubule filaments [24,25]. FAK aggravates cancer progression by activating the Src, PI3K-Akt, and Raf-MEK-ERK signaling pathways [26], and the deletion of FAK in mam- 
mary epithelial cells suppresses tumor formation and progression in mouse models of breast cancer [27-29]. JNKs are MAPK family enzymes that are best known for regulating proapoptotic signaling and transcriptional activation, but they are also involved in the expression of EMT markers and matrix metalloproteinases via the regulation of c-Jun and AP-1, which stimulate cell motility [30-32]. In line with these observations, BHMPS treatment or silencing of Rab27a significantly decreased FAK and JNK activation, with a reduction in the levels of EMT-activating transcription factors.

Rab27a has been reported to regulate vesicle transport and exocytosis by acting as a compartment-specific molecular switch [4,33]. Rab27 consists of two isoforms, Rab27a and Rab27b, which share high sequence similarity (71\% amino acid identity) but function differently even in the same cell type [34,35]. For example, knockdown of Rab27a (or its potential effector Slp4) causes a decrease in the number of plasma membrane-docked multivesicular endosomes (MVEs) and increased sizes of MVEs, whereas knockdown of Rab27b (or Slac2-b) causes perinuclear clustering of MVEs and a decrease in the size of the MVEs in HeLa cells [36]. Moreover, only Rab27a, not Rab27b, is required for exosome secretion by murine mammary carcinoma cells [37]. Several studies have shown that Rab27a plays a crucial role in exosome secretion, such as insulin secretion by pancreatic $\beta$-cells, melanosome transport by melanocytes, cytotoxic granule exocytosis by immune cells, and reactive oxygen species (ROS) production by macrophages and neutrophils $[6,35,37,38]$. Although the contents of exosomes were not identified owing to experimental limitations in this study, the inhibition of Rab27a expression significantly decreased the secretion of oncogenic EGFR and the ECM protein FN. Because EGFR is frequently overexpressed in TNBC than in normal tissue and contributes to malignancy and invasiveness [39,40], Rab27a may be a promising target to disrupt these secretory pathways. However, since there are more than 65 Rab protein subfamilies and several factors related to Rab proteins, including effectors, binding proteins, and regulators, further studies are required to determine whether the interaction between Slp4 and Rab27a has sufficient specificity and selectivity to be responsible for Rab27a-mediated vesicle biogenesis and trafficking.

Several studies have demonstrated that Rab27a blockade decreased primary tumor growth and the number of metastatic lung foci in $4 \mathrm{~T} 1$ cell-transplanted mice but not in TS/A cell-injected mice [12,37]. Additionally, upregulation of Rab27a expression promotes rapid growth of primary tumors and increases metastatic potential [7,12]. However, we found that Rab27a-overexpressing MDA-MB-231 xenograft tumors grew significantly slower than control tumors, and the anticancer activity of BHMPS was only observed in Rab27a-overexpressing tumor xenografts. A recent study reported that ionizing radiation accelerates the exosome secretion pathway by upregulating Rab27a and Rab27b levels in MCF-7 cells, thereby increasing ROS production and apoptosis [41]. This finding suggests the possibility that Rab27a-mediated MVE secretion may have a detrimental effect not only on the secretory cells themselves but also on adjacent cells of the TME. Moreover, since accumulation of neutrophils in tumor-bearing mice modifies TME by secretion of factors that promote angiogenesis and increase tumor cell migration [33,42,43], the significant decrease of neutrophils in Rab27a-overexpressing tumor-bearing mice in this study may be another plausible explanation for the unexpected growth inhibition of Rab27aoverexpressing tumors. It has been documented that neutrophils are the predominant circulating leukocyte population in humans and tumor-associated neutrophils and their myeloid precursors in the spleen, bone marrow, and blood play an important role in cancer biology [44]. Furthermore, neutrophil infiltration has been associated with poor prognosis in some cancer patients, but further investigation into the polarization, conversion, and function regulation of $\mathrm{N} 1$ or $\mathrm{N} 2$ is required. We speculate that the significant decrease in neutrophils in Rab27a-overexpressing tumor-bearing mice may be attributed to the secretory substances that reduce the number of neutrophils or inhibit neutrophil attraction. Secreted molecules may vary depending on stimuli, the binding partners of Rab27a, specific cell types, etc. For example, endogenous Rab27a may produce tumor-promoting factors, while excess Rab27a may additionally release more toxic and cytocidal factors. Future 
studies should be directed towards examining these possibilities to further dissect the mechanism involved.

In contrast to the observation that Rab27a expression was upregulated in clinical cancer patients $[45,46]$, our analysis using public databases showed different correlations between mRNA and protein levels. There is a considerably greater shortage of publicly available proteome databases than transcriptome databases such as TCGA, and using datasets with different data often produces different results. Interestingly, it has been reported that the evident discrepancies between transcript and protein levels, such as groups with high protein levels but low transcript levels, were mostly associated with vesicle-mediated transport. The negative correlation may be explained by protein transport and spatial separation [47]. According to a recent meta-analysis evaluating the prognostic significance of Rab27 expression in solid tumors, poor survival was significantly associated with high Rab27b expression but not with high Rab27a expression [48]. Moreover, Shi et al. reported that high expression of Rab27a indicates a favorable prognosis, contrary to other studies [49]. Therefore, more thorough validation and a careful approach will be required to apply Rab27a as a promising prognostic marker and as a druggable target.

There are some limitations in the present study that should be addressed. First, the involvement of other Rab27 families in breast cancer invasion and metastasis remains to be elucidated, particularly with regard to how cells select Rab27a among approximately $70 \mathrm{Rab}$ families. Second, it is necessary to determine the contents loaded into exosomes, which can expand the application of Rab27a as a valuable biomarker. Accordingly, the binding partners of Rab27a need to be identified, and whether they are changeable depending on the environment also needs to be determined. Third, it should be validated whether Rab27a is a critical factor in breast cancer progression and/or metastasis. In this case, the similarities and differences of Rab27a-mediated exocytosis between immune cells and in cancer should be established. Lastly, further investigation is needed to determine whether off-target effects of BHMPS exist. These will advance our fundamental understanding of Rab27a-mediated exocytosis and metastasis.

\section{Materials and Methods}

\subsection{Materials}

Antibodies and chemicals are listed in the Supplementary Table S1 for vendor and catalog numbers. BHMPS $\left(\mathrm{C}_{20} \mathrm{H}_{20} \mathrm{~N}_{4} \mathrm{O}_{4} \mathrm{~S}\right.$, MW: 412.46) was synthesized using a previously reported method [14] or purchased from 4chem laboratory (Suwon, Gyeonggi-do, Korea).

\subsection{Cell Cultures}

Human breast cancer cells, MDA-MB-231, BT549, BT474, and MCF7 were obtained from the American Type Culture Collection (ATCC, Manassas, VA, USA). BT549 cells were cultured in RPMI 1640 supplemented with 10\% FBS, penicillin (100 units $/ \mathrm{mL}$ ), and streptomycin $(100 \mu \mathrm{g} / \mathrm{mL})$. MDA-MB-231 and BT474 cells were grown in DMEM supplemented with 10\% FBS, penicillin (100 units $/ \mathrm{mL})$, and streptomycin $(100 \mu \mathrm{g} / \mathrm{mL})$. MCF7 cells were maintained in MEM supplemented with 10\% FBS, penicillin (100 units $/ \mathrm{mL}$ ), and streptomycin $(100 \mu \mathrm{g} / \mathrm{mL})$. Cells were incubated in a humidified incubator at $37^{\circ} \mathrm{C}$ in a $5 \%$ $\mathrm{CO}_{2}$ atmosphere.

\subsection{Cell Viability Assay}

Cancer cells were seeded in 96-well plates $\left(1 \times 10^{4}\right.$ cells/well). The cells were treated with two-fold serial dilutions of BHMPS from $100 \mu \mathrm{M}$ to $0.4 \mu \mathrm{M}$ for $24 \mathrm{~h}$ and further incubated with MTT dye solution $(0.5 \mathrm{mg} / \mathrm{mL})$ for $4 \mathrm{~h}$. The formed formazan crystals were dissolved in DMSO, and the absorbance was measured using a microplate reader at $550 \mathrm{~nm}$. 


\subsection{Migration Assay}

Cells were seeded in 6 -well plates $\left(5 \times 10^{5}\right.$ cells/well). After $24 \mathrm{~h}$, the cells were scratched using a $200 \mu \mathrm{L}$ pipette tip, followed by the administration of $10 \mu \mathrm{M}$ BHMPS. The migrated cells were stained with $0.05 \%$ crystal violet, photographed at $40 \times$ magnification, and analyzed under a microscope (Olympus IX73; Olympus, Tokyo, Japan). The data taken were the distance between the two migrated surfaces of cells coming from either side of the wounded area.

\subsection{Invasion Assay}

For the Matrigel invasion assay, cells $\left(5 \times 10^{4}\right.$ cells / well $)$ in serum-free medium were loaded into the upper well of Matrigel-coated Transwell chamber with $8 \mu \mathrm{m}$ pore size (Corning Inc., Corning, NY, USA), while the lower well was filled with culture medium. After $24 \mathrm{~h}$, the cells that migrated to the lower surface of the membrane were counted in 10 microscopic fields per well, and the average value was quantified.

\subsection{Western Blot Analysis}

Cells were lysed using buffer containing $50 \mathrm{mM}$ Tris- $\mathrm{HCl}(\mathrm{pH} 7.4), 1 \%$ NP-40, $150 \mathrm{mM}$ $\mathrm{NaCl}, 1 \mathrm{mM}$ EDTA, $1 \mathrm{mM}$ PMSF, $1 \mu \mathrm{g} / \mathrm{mL}$ aprotinin, $1 \mathrm{mM} \mathrm{Na} \mathrm{VO}_{4}$, and $1 \mathrm{mM} \mathrm{NaF}$. Proteins were separated using SDS-PAGE and transferred onto nitrocellulose membranes (GE Healthcare Life Science, Little Chalfont, UK). Proteins were analyzed using the specified antibodies and an ECL detection system (GE Healthcare). ImageJ software version $1.53 \mathrm{~h}$ (National Institutes of Health, Bethesda, MD, USA) was used for analysis. Representative images from three or more experiments are shown.

\subsection{Transfection}

Cells were transfected with plasmids and/or small interfering RNA (siRNA) (20 nM) using Lipofectamine 2000 or Lipofectamine RNAiMAX (Invitrogen, Carlsbad, CA, USA) according to the manufacturer's protocol. For the establishment of stable cell, MDA-MB-231 cells were transfected with pCMV6-Rab27a-Myc-DDK expression vector (\#RC217900; OriGene, Rockville, MD, USA) and supplied with $1000 \mu \mathrm{g} / \mathrm{mL}$ of G418 (\#ant-gn-1; InvivoGen, San Diego, CA, USA)-containing media. Visible individual colonies were transferred into multi-well plates, after which stably expressing cells were selected using qPCR and western blot analysis. A B6 clone was finally obtained.

\subsection{Immunoprecipitation}

Cell lysates were used for immunoprecipitation with magnetic bead-conjugated MycTag antibodies according to the manufacturer's protocol. Immunoprecipitated proteins were subjected to SDS-PAGE using the specified antibody. Negative-control experiments were performed in the same way using normal IgG and magnetic beads with the same amount of protein.

\subsection{Immunofluorescence Confocal Microscopy}

Paraformaldehyde-fixed cells were incubated with the indicated primary and fluorescent dye-conjugated secondary antibodies (Invitrogen). Slides were mounted using Duolink Mounting Media with DAPI and images were acquired under an LSM880 confocal microscope (Zeiss, Jena, Germany) using the Zen 2.3 software (Zeiss) for image processing and analysis. Representative images are shown from three or more independent experiments.

\subsection{Proximity Ligation Assay (PLA)}

Direct protein-protein interactions were visualized using in situ PLA [50] using Duolink In Situ Red Starter Kit Mouse/Rabbit (Sigma-Aldrich). After $24 \mathrm{~h}$ incubation of cells treated with serum and/or BHMPS, the cells were fixed, permeabilized, blocked, and co-incubated with anti-Rab27a and anti-Slp4 primary antibodies overnight. The next day, Duolink anti-rabbit PLUS and anti-mouse MINUS secondary antibodies and the red 
detection reagent were treated. After amplification of PLA signals, images were acquired under an LSM880 confocal microscope (Zeiss) and were analyzed with the Zen 2.3 software (Zeiss).

\subsection{Tumor Xenograft Mouse Model}

Six-week-old female C57BL/ 6 and athymic BALB/c nude mice (Orient Bio Inc., Seongnam, Korea) were housed under specific pathogen-free conditions in microisolator cages and supplied laboratory chow and water ad libitum. In the experimental lung metastasis model, B16F10 cells $\left(1 \times 10^{6}\right.$ cells $/ 100 \mu \mathrm{L}$ phosphate-buffered saline (PBS) $)$ were injected intravenously into the tail vein of C57BL/ 6 mice, and BHMPS was administered six times every $2 \mathrm{~d}$ starting from the 3rd day. The mice were sacrificed, and the lungs were harvested after $20 \mathrm{~d}$ of tumor cell injection. In the xenograft model, MDA-MB-231 cells and MDAMB-231 cells stably overexpressing Rab27a ( $2 \times 10^{6}$ cells) in $0.1 \mathrm{~mL}$ PBS with 50\% Matrigel were injected subcutaneously into the thigh hind leg of BALB/c nude mice. Tumors were measured along two axes (L, longest axis; W, shortest axis) with a Vernier caliper (Mitutoyo, Kawasaki, Japan) two or three times a week, and the tumor volume was calculated as $\left[\left(\mathrm{L} \times \mathrm{W}^{2}\right) / 2\left(\mathrm{~mm}^{3}\right)\right]$. When the tumor volumes reached approximately $150 \mathrm{~mm}^{3}$, the mice were treated with BHMPS (10 mg/kg of bodyweight) every $2 \mathrm{~d}$ for a total of 10 times. These experiments were reviewed and approved by the Institutional Animal Care and Use Committee of the Korea Institute of Radiological and Medical Sciences (KIRAMS 2020-0036).

\subsection{Bioinformatics Analysis}

To analyze the clinical significance of Rab27a expression levels, The Cancer Genome Atlas (TCGA) data were investigated using the TIMER2.0 database (http:/ / timer.cistrome. org /) (accessed on 2 July 2021). The overall survival (OS) according to Rab27a mRNA and protein expression in breast cancer were analyzed using the Kaplan-Meier Plotter (https: / / kmplot.com/analysis /) (accessed on 18 June 2021). The patient group was split using the median value, and the result of the dataset with the lowest $p$-value for each analysis was represented. The UALCAN website (http:/ / ualcan.path.uab.edu/) (accessed on 5 July 2021) was used to analyze Rab27a expression in major subclasses and at different stages of breast cancer at the mRNA and protein levels.

\subsection{Data Analysis}

Data were presented as mean \pm standard deviation (SD) except for the mouse model experiments expressed as mean \pm standard error (SE). Statistical differences between groups were analyzed by Student's $t$-test (two-tailed) or analysis of variance (ANOVA) using GraphPad Prism software version 9.0 (GraphPad, La Jolla, CA, USA). p-values lower than 0.05 were considered statistically significant.

\section{Conclusions}

In conclusion, our findings demonstrated for the first time that BHMPS regulates the secretory process of intracellular vesicles by disrupting the interaction between Rab27a and Slp4 in breast cancer cells. FAK and JNK activation regulate Rab27a-mediated exocytosis for the secretion of EGFR and FN, which facilitate migration and invasion. This study also highlighted the importance of understanding the mechanisms of Rab27a-mediated metastasis, which are still largely unknown, in improving the therapeutic options for metastatic cancers.

Supplementary Materials: The following supporting information can be downloaded at: https: //www.mdpi.com/article/10.3390/cancers14020373/s1, Figure S1: The expression of Rab27a in human tumor and adjacent normal tissues; Table S1: List of details for the antibodies and chemicals. File S1: Full Western blot images. 
Author Contributions: Study design: J.-I.P., J.K. (Joon Kim), S.-Y.J. and J.-Y.S.; Study conception: J.A., J.-K.P., J.K. (Jaesung Kim) and J.-Y.S.; Chemical design and synthesis: H.K.C.; All biological experiments and data analysis: J.-I.P., J.L., S.-M.K., S.-J.C. and S.-Y.J.; Data discussion: K.-H.S., S.-G.H., D.-S.L., S.-Y.J. and J.-Y.S.; Manuscript writing: J.-I.P., S.-Y.J. and J.-Y.S. All authors have read and agreed to the published version of the manuscript.

Funding: This study was supported by the National Research Foundation of Korea (NRF-2020R1A2C1 007138 and NRF-2020M2D9A2094153) and the Korea Institute of Radiological and Medical Sciences (50538-2021) funded by the Korean government, Ministry of Science and ICT.

Institutional Review Board Statement: All animal experiments were approved by the Institutional Animal Care and Use Committee of the Korea Institute of Radiological and Medical Sciences and are reported in accordance with the ARRIVE (Animal Research: Reporting of In Vivo Experiments) guidelines (kirams 2020-0036, 9 June 2020).

Informed Consent Statement: Not applicable.

Data Availability Statement: All other relevant data are available from the corresponding author upon reasonable request.

Conflicts of Interest: The authors declare that they have no conflict of interest.

\begin{abstract}
Abbreviation
BHMPS, (E)-N-benzyl-6-(2-(3, 4-dihydroxybenzylidene) hydrazinyl)- $N$-methylpyridine-3-sulfonamide; EMT, epithelial-mesenchymal transition; EGFR, epidermal growth factor receptor; FAK, focal adhesion kinase; JNK, c-Jun N-terminal kinase; siRNA, small interfering RNA; Slp, synaptotagmin-like protein; PLA, proximity ligation assay.
\end{abstract}

\title{
References
}

1. Fares, J.; Fares, M.Y.; Khachfe, H.H.; Salhab, H.A.; Fares, Y. Molecular principles of metastasis: A hallmark of cancer revisited. Signal Transduct Target Ther. 2020, 5, 28. [CrossRef]

2. Kalluri, R.; Weinberg, R.A. The basics of epithelial-mesenchymal transition. J. Clin. Investig. 2009, 119, 1420-1428. [CrossRef] [PubMed]

3. Recchi, C.; Seabra, M.C. Novel functions for Rab GTPases in multiple aspects of tumour progression. Biochem. Soc. Trans. 2012, 40, 1398-1403. [CrossRef]

4. Blanc, L.; Vidal, M. New insights into the function of Rab GTPases in the context of exosomal secretion. Small GTPases 2018, 9 , 95-106. [CrossRef] [PubMed]

5. Nagashima, K.; Torii, S.; Yi, Z.; Igarashi, M.; Okamoto, K.; Takeuchi, T.; Izumi, T. Melanophilin directly links Rab27a and myosin Va through its distinct coiled-coil regions. FEBS Lett. 2002, 517, 233-238. [CrossRef]

6. Yi, Z.; Yokota, H.; Torii, S.; Aoki, T.; Hosaka, M.; Zhao, S.; Takata, K.; Takeuchi, T.; Izumi, T. The Rab27a/granuphilin complex regulates the exocytosis of insulin-containing dense-core granules. Mol. Cell Biol. 2002, 22, 1858-1867. [CrossRef] [PubMed]

7. Peinado, H.; Aleckovic, M.; Lavotshkin, S.; Matei, I.; Costa-Silva, B.; Moreno-Bueno, G.; Hergueta-Redondo, M.; Williams, C.; Garcia-Santos, G.; Ghajar, C.; et al. Melanoma exosomes educate bone marrow progenitor cells toward a pro-metastatic phenotype through MET. Nat. Med. 2012, 18, 883-891. [CrossRef] [PubMed]

8. Yuan, X.; Qian, N.; Ling, S.; Li, Y.; Sun, W.; Li, J.; Du, R.; Zhong, G.; Liu, C.; Yu, G.; et al. Breast cancer exosomes contribute to pre-metastatic niche formation and promote bone metastasis of tumor cells. Theranostics 2021, 11, 1429-1445. [CrossRef]

9. Dai, J.; Su, Y.; Zhong, S.; Cong, L.; Liu, B.; Yang, J.; Tao, Y.; He, Z.; Chen, C.; Jiang, Y. Exosomes: Key players in cancer and potential therapeutic strategy. Signal Transduct Target Ther. 2020, 5, 145. [CrossRef] [PubMed]

10. Guo, L.; Guo, N. Exosomes: Potent regulators of tumor malignancy and potential bio-tools in clinical application. Crit. Rev. Oncol. Hematol. 2015, 95, 346-358. [CrossRef]

11. Wu, Q.; Zhou, L.; Lv, D.; Zhu, X.; Tang, H. Exosome-mediated communication in the tumor microenvironment contributes to hepatocellular carcinoma development and progression. J. Hematol. Oncol. 2019, 12, 53. [CrossRef]

12. Li, Z.; Fang, R.; Fang, J.; He, S.; Liu, T. Functional implications of Rab27 GTPases in Cancer. Cell. Commun. Signal 2018, 16, 44. [CrossRef]

13. Joung, J.Y.; Lee, H.Y.; Park, J.; Lee, J.Y.; Chang, B.H.; No, K.T.; Nam, K.Y.; Hwang, J.S. Identification of novel rab27a/melanophilin blockers by pharmacophore-based virtual screening. Appl. Biochem. Biotechnol. 2014, 172, 1882-1897. [CrossRef]

14. Kang, S.M.; Nam, K.Y.; Jung, S.Y.; Song, K.H.; Kho, S.; No, K.T.; Choi, H.K.; Song, J.Y. Inhibition of cancer cell invasion by new ((3,4-dihydroxy benzylidene)hydrazinyl)pyridine-3-sulfonamide analogs. Bioorg. Med. Chem. Lett. 2016, 26, 1322-1328. [CrossRef]

15. De Craene, B.; Berx, G. Regulatory networks defining EMT during cancer initiation and progression. Nat. Rev. Cancer 2013, 13, 97-110. [CrossRef] 
16. Braicu, C.; Buse, M.; Busuioc, C.; Drula, R.; Gulei, D.; Raduly, L.; Rusu, A.; Irimie, A.; Atanasov, A.G.; Slaby, O.; et al. A Comprehensive Review on MAPK: A Promising Therapeutic Target in Cancer. Cancers 2019, 11, 1618. [CrossRef] [PubMed]

17. McLean, G.W.; Carragher, N.O.; Avizienyte, E.; Evans, J.; Brunton, V.G.; Frame, M.C. The role of focal-adhesion kinase in cancer-A new therapeutic opportunity. Nat. Rev. Cancer 2005, 5, 505-515. [CrossRef] [PubMed]

18. Fukuda, M. Rab27 effectors, pleiotropic regulators in secretory pathways. Traffic 2013, 14, 949-963. [CrossRef] [PubMed]

19. Wang, J.S.; Wang, F.B.; Zhang, Q.G.; Shen, Z.Z.; Shao, Z.M. Enhanced expression of Rab27A gene by breast cancer cells promoting invasiveness and the metastasis potential by secretion of insulin-like growth factor-II. Mol. Cancer Res. 2008, 6, 372-382. [CrossRef]

20. Hessvik, N.P.; Llorente, A. Current knowledge on exosome biogenesis and release. Cell Mol. Life Sci. 2018, 75, 193-208. [CrossRef]

21. Huang, T.; Deng, C.X. Current Progresses of Exosomes as Cancer Diagnostic and Prognostic Biomarkers. Int. J. Biol. Sci. 2019, 15, 1-11. [CrossRef]

22. Schlaepfer, D.D.; Mitra, S.K. Multiple connections link FAK to cell motility and invasion. Curr. Opin. Genet. Dev. 2004, 14, 92-101. [CrossRef]

23. Zhao, J.; Guan, J.L. Signal transduction by focal adhesion kinase in cancer. Cancer Metastasis Rev. 2009, 28, 35-49. [CrossRef]

24. De Franceschi, N.; Hamidi, H.; Alanko, J.; Sahgal, P.; Ivaska, J. Integrin traffic-The update. J. Cell Sci. 2015, 128, 839-852. [CrossRef] [PubMed]

25. Alanko, J.; Ivaska, J. Endosomes: Emerging Platforms for Integrin-Mediated FAK Signalling. Trends Cell Biol 2016, 26, 391-398 [CrossRef] [PubMed]

26. Harburger, D.S.; Calderwood, D.A. Integrin signalling at a glance. J. Cell Sci. 2009, 122 Pt 2, 159-163. [CrossRef]

27. Luo, M.; Fan, H.; Nagy, T.; Wei, H.; Wang, C.; Liu, S.; Wicha, M.S.; Guan, J.L. Mammary epithelial-specific ablation of the focal adhesion kinase suppresses mammary tumorigenesis by affecting mammary cancer stem/progenitor cells. Cancer Res. 2009, 69, 466-474. [CrossRef] [PubMed]

28. Provenzano, P.P.; Inman, D.R.; Eliceiri, K.W.; Beggs, H.E.; Keely, P.J. Mammary epithelial-specific disruption of focal adhesion kinase retards tumor formation and metastasis in a transgenic mouse model of human breast cancer. Am. J. Pathol. 2008, 173, 1551-1565. [CrossRef]

29. Pylayeva, Y.; Gillen, K.M.; Gerald, W.; Beggs, H.E.; Reichardt, L.F.; Giancotti, F.G. Ras- and PI3K-dependent breast tumorigenesis in mice and humans requires focal adhesion kinase signaling. J. Clin. Investig. 2009, 119, 252-266. [CrossRef]

30. Ulu, A.; Frost, J.A. Regulation of RhoA activation and cell motility by c-Jun N-terminal kinases and Net1. Small GTPases 2020, 11, 385-391. [CrossRef]

31. Cheng, H.L.; Lin, C.W.; Yang, J.S.; Hsieh, M.J.; Yang, S.F.; Lu, K.H. Zoledronate blocks geranylgeranylation not farnesylation to suppress human osteosarcoma U2OS cells metastasis by EMT via Rho A activation and FAK-inhibited JNK and p38 pathways Oncotarget 2016, 7, 9742-9758. [CrossRef]

32. Wang, J.F.; Zhao, K.; Chen, Y.Y.; Qiu, Y.; Zhu, J.H.; Li, B.P.; Wang, Z.; Chen, J.Q. NKCC1 promotes proliferation, invasion and migration in human gastric cancer cells via activation of the MAPK-JNK/EMT signaling pathway. J. Cancer 2021, 12, 253-263. [CrossRef]

33. Miller, I.V.; Grunewald, T.G.P. Tumour-derived exosomes: Tiny envelopes for big stories. Biol. Cell 2015, 107, 287-305. [CrossRef] [PubMed]

34. Singh, R.K.; Mizuno, K.; Wasmeier, C.; Wavre-Shapton, S.T.; Recchi, C.; Catz, S.D.; Futter, C.; Tolmachova, T.; Hume, A.N.; Seabra, M.C. Distinct and opposing roles for Rab27a/Mlph/MyoVa and Rab27b/Munc13-4 in mast cell secretion. FEBS J. 2013, 280, 892-903. [CrossRef] [PubMed]

35. Johnson, J.L.; Brzezinska, A.A.; Tolmachova, T.; Munafo, D.B.; Ellis, B.A.; Seabra, M.C.; Hong, H.; Catz, S.D. Rab27a and Rab27b regulate neutrophil azurophilic granule exocytosis and NADPH oxidase activity by independent mechanisms. Traffic 2010, 11, 533-547. [CrossRef] [PubMed]

36. Ostrowski, M.; Carmo, N.B.; Krumeich, S.; Fanget, I.; Raposo, G.; Savina, A.; Moita, C.F.; Schauer, K.; Hume, A.N.; Freitas, R.P.; et al. Rab27a and Rab27b control different steps of the exosome secretion pathway. Nat. Cell Biol. 2010, 12, 19-30. [CrossRef] [PubMed]

37. Bobrie, A.; Krumeich, S.; Reyal, F.; Recchi, C.; Moita, L.F.; Seabra, M.C.; Ostrowski, M.; Thery, C. Rab27a supports exosomedependent and -independent mechanisms that modify the tumor microenvironment and can promote tumor progression. Cancer Res. 2012, 72, 4920-4930. [CrossRef]

38. Guo, D.; Lui, G.Y.L.; Lai, S.L.; Wilmott, J.S.; Tikoo, S.; Jackett, L.A.; Quek, C.; Brown, D.L.; Sharp, D.M.; Kwan, R.Y.Q.; et al. RAB27A promotes melanoma cell invasion and metastasis via regulation of pro-invasive exosomes. Int. J. Cancer 2019, 144, 3070-3085. [CrossRef]

39. Song, X.; Liu, Z.; Yu, Z. EGFR Promotes the Development of Triple Negative Breast Cancer Through JAK/STAT3 Signaling Cancer Manag. Res. 2020, 12, 703-717. [CrossRef]

40. Corkery, B.; Crown, J.; Clynes, M.; O'Donovan, N. Epidermal growth factor receptor as a potential therapeutic target in triple-negative breast cancer. Ann. Oncol. 2009, 20, 862-867. [CrossRef]

41. Jabbari, N.; Nawaz, M.; Rezaie, J. Ionizing Radiation Increases the Activity of Exosomal Secretory Pathway in MCF-7 Human Breast Cancer Cells: A Possible Way to Communicate Resistance against Radiotherapy. Int. J. Mol. Sci. 2019, 20, 3649. [CrossRef]

42. Wang, M.; Zhao, J.; Zhang, L.; Wei, F.; Lian, Y.; Wu, Y.; Gong, Z.; Zhang, S.; Zhou, J.; Cao, K.; et al. Role of tumor microenvironment in tumorigenesis. J. Cancer 2017, 8, 761-773. [CrossRef] [PubMed] 
43. Soysal, S.D.; Tzankov, A.; Muenst, S.E. Role of the Tumor Microenvironment in Breast Cancer. Pathobiology 2015, 82, 142-152. [CrossRef] [PubMed]

44. Fridlender, Z.G.; Albelda, S.M. Tumor-associated neutrophils: Friend or foe? Carcinogenesis 2012, 33, 949-955. [CrossRef] [PubMed]

45. Akavia, U.D.; Litvin, O.; Kim, J.; Sanchez-Garcia, F.; Kotliar, D.; Causton, H.C.; Pochanard, P.; Mozes, E.; Garraway, L.A.; Pe'er, D. An integrated approach to uncover drivers of cancer. Cell 2010, 143, 1005-1017. [CrossRef] [PubMed]

46. Dong, W.W.; Mou, Q.; Chen, J.; Cui, J.T.; Li, W.M.; Xiao, W.H. Differential expression of Rab27A/B correlates with clinical outcome in hepatocellular carcinoma. World J. Gastroenterol. 2012, 18, 1806-1813. [CrossRef] [PubMed]

47. Moritz, C.P.; Muhlhaus, T.; Tenzer, S.; Schulenborg, T.; Friauf, E. Poor transcript-protein correlation in the brain: Negatively correlating gene products reveal neuronal polarity as a potential cause. J. Neurochem. 2019, 149, 582-604. [CrossRef]

48. Koh, H.M.; Jang, B.G.; Kim, D.C. Prognostic significance of Rab27 expression in solid cancer: A systematic review and metaanalysis. Sci. Rep. 2020, 10, 14136. [CrossRef]

49. Shi, C.; Yang, X.; Ni, Y.; Hou, N.; Xu, L.; Zhan, F.; Zhu, H.; Xiong, L.; Chen, P. High Rab27A expression indicates favorable prognosis in CRC. Diagn. Pathol. 2015, 10, 68. [CrossRef]

50. Koos, B.; Andersson, L.; Clausson, C.M.; Grannas, K.; Klaesson, A.; Cane, G.; Soderberg, O. Analysis of protein interactions in situ by proximity ligation assays. Curr. Top. Microbiol. Immunol. 2014, 377, 111-126. 